\title{
¿Dar Cabida o Hacer Parte? Significados de la Participación en Dos Políticas Inclusivas Chilenas
}

\author{
Making Room for or Getting Involved into? Meanings of \\ Participation among Two Chilean Inclusive Policies
}

\author{
Claudio Herrera * \\ Universidad Bernardo O’Higgins, Chile
}

\begin{abstract}
Este artículo presenta el análisis realizado a dos políticas educativas chilenas relativas a la inclusión -El Decreto 170/2010 que "Fija normas para determinar los alumnos con Necesidades Educativas Especiales que serán beneficiarios de las subvenciones para Educación Especial” y el Decreto 83/2015 que “Aprueba criterios y orientaciones de Adecuación Curricular para estudiantes con Necesidades Educativas Especiales de Educación Parvularia y Educación Básica”- con el objetivo de identificar los significados que adquiere la participación como modalidad inclusiva dentro del texto. Utilizando algunos procedimientos del Análisis Crítico del Discurso emergen, dentro del conjunto de las políticas consideradas, operaciones discursivas que designan las jerarquías, relevancias y énfasis que la participación toma dentro de los objetivos que la política inclusiva prescribe e instituye. Asimismo, desde los resultados se corrobora una relegación de la noción de participación hacia un lugar destituido de su pretensión original dinamizadora del proceso inclusivo, y sustituida por marcadores que la desvinculan de la relación de interdependencia que esta constituye, por ejemplo, con el aprendizaje. Esta posición adquiere particular relevancia cuando se consideran los parámetros que la política educativa, como parte de las racionalidades que entran en juego en su diseño, termina programando en relación a la inclusión como horizonte democrático.
\end{abstract}

Descriptores: Inclusión; Política pública; Participación; Discurso; Educación.

This article presents the discourse analysis of two inclusive-education Chilean policies - the Decree 170/2010 which "Establishes several criteria in order to determine whether students with Special Educational Needs are Special Education grant eligibility" and Decree 83/2015 which "Approves Curriculum Adaptation criteria and orientations for Special Educational Needs pre-school and elementary school students" - in order to identify the specific meanings of participation as the inclusive modality takes within both of theme and considered part of a whole inclusive discourse. Based on some principles and procedures given by the Critical Discourse Analysis (CDA) - a set of analysis techniques, inclusive policies address discursive operations that distribute hierarchies, relevancies and focuses that reframes the participation idea which ends up embodying based on what inclusive policy finally remarks in this case. Furthermore, results underline a destitution operation that relegates participation to a secondary place, taking away and replacing its original meaning - the one that moves the inclusive process alongside with other educative key criteria such as learning. This position and outcome gets particular relevance when inclusive parameters are taken into account, as a part of a rational speech embodying inclusion within school debates, conditioning what this aspiration addresses as a democratic horizon in Chilean educational policy.

Keywords: Inclusion; Public policy; Participation; Discourse; Education.

*Contacto: claudioherrera@docente.ubo.cl

Recibido: 


\section{Introducción}

Cada vez con mayor fuerza, en Chile ha tomado forma una intensa discusión sobre las mejores alternativas para reducir las brechas de desigualdad en sus distintos escenarios sociales. Alineada con este debate, la articulación estatal por alcanzar mejores condiciones de vida para todos los sujetos (OECD, 2018) ha adquirido una importancia paradigmática. De la mano de una serie de reformas políticas de acceso y universalización de derechos en áreas como trabajo, salud y educación, el Estado chileno ha ratificado su interés por impulsar agendas cuyas propuestas programáticas se muestren consistentes con el esfuerzo mundial por conseguir sociedades más integradas, justas y equitativas (Ministerio de Educación, 2016). En sintonía con una intención que ha visto, en las eventuales transformaciones del campo educativo, un ámbito protagónico que ofrecería posibilidades viables para la construcción de proyectos tendientes a forjar sociedades más igualitarias (Popkewitz, 2018), buena parte de las principales innovaciones de la política educativa nacional se han orientado desde dicha pretensión. En particular, situados al interior de la construcción de este novedoso andamiaje, la propuesta estatal ha sido configurada ampliamente a partir de la noción de inclusión (MINEDUC, 2015). La centralidad de dicha idea, en tanto caracterizaría una política estatal novedosa y pertinente, aglutina en sí misma cierto valor democrático que asume, además, como concreta la tentativa que el Estado tiene de objetivar una aspiración programática y susceptible de ser declarada como principio normativo (Echeita, 2006). Tomando en cuenta dicha pretensión institucional, la noción de inclusión se vincula principalmente con el reconocimiento y la visibilización de una heterogeneidad estudiantil que antes orbitaba por fuera de los límites de lo típicamente educable.

En consecuencia, las repuestas habituales que se han levantado en torno a tal aspiración han venido fundamentadas por una idea de inclusión que es sostenida, además de norma, como atributo inherente: pues entiende la escolarización como una garantía que es conferida al sujeto. En otras palabras, esta última dimensión se plantea como una prerrogativa ratificada como derecho, que debe instar al sistema a generar condiciones de educabilidad para la inclusión de la población escolar completa al interior de los centros educativos.

En principio fortalecida por las directrices que se inician con la Convención sobre los Derechos del Niño (1989), y continuada después por las iniciativas de reivindicación escolar del movimiento internacional Educación para Todos (UNESCO, 1990), la consideración por la diversidad dentro de la escuela se consolida en las institucionalidades estatales a finales de siglo a partir de la necesidad de generar, en el marco de acuerdos educativos ratificados por los gobiernos, ordenamientos que sean capaces de ofrecer, reconocer y hacer posible condiciones que allanen este objetivo de pensar el abordaje de la diversidad como una lineamiento democratizador y programático viable, que en la práctica sea capaz de generar contextos que incluyan, reciban y hagan convivir al estudiantado.

Desde esta perspectiva, la inclusión es una aspiración que surge, además, desde la necesidad de situar algunas condiciones contextuales y entramados institucionales como participantes directos y determinantes del fenómeno de la segregación educativa (Ainscow, 2016). Abordar dicho problema, entonces, involucra determinar, apuntalar y reconocer la convivencia de comunidades heterogéneas que dialogan y aprenden desde sus particularidades (MINEDUC, 2016). Por lo tanto, la inclusión como propósito 
comportaría, ante todo, una narrativa correctiva de la escuela como lugar de encuentro (MINEDUC, 2013).

Ahora bien, en plena concordancia con los términos y garantías bajo los cuales se organiza dicha propuesta educativa, uno de los programas inclusivos que ha gozado de mayor impacto programático en la política inclusiva, y que a la fecha suscita el mayor consenso en torno a su aplicación y pertinencia práctica (Mendía, 2012), es la sistematización que Booth y Ainscow (2012) han propuesto en el Index for Inclusion: Developing Learning an Participation in Schools (en adelante Index for inclusion), matriz operacional que, entre otras cosas, inspira, articula y condiciona gran parte del diseño que la política nacional, como se planteaba, considera como parámetro organizador en materia de educación en general y de atención a la diversidad en particular (MINEDUC, 2019). Desde este punto de vista, la aplicabilidad del Index for inclusion -desarrollado en Inglaterra a principios de siglo- se configura como un instrumento esencial para fomentar procesos de cambio educativo que tengan como horizonte la inclusión educativa (Booth et al., 2002; Sandoval et al., 2002).

Dentro de la conceptualización que elabora el Index for inclusion para tipificar aquello que entiende por inclusión, cobran especial relevancia los términos en los que se plantea metodológicamente el proceso inclusivo. En este sentido, la aspiración concreta que se asume es contribuir a aumentar, cuanto sea posible, las posibilidades de aprendizaje y participación dentro de las escuelas que utilicen el instrumento para implementar procesos de mejora (Booth et al., 2015).

Asimismo, tomando en cuenta el proceso normativo articulado desde el Index for inclusion, y que la institucionalidad programática ofrece bajo un marco legal que estipula que incluye, existe evidencia que documenta - en la evaluación de las modalidades de implementación de algunas políticas inclusivas nacionales- dificultades en torno a articular, reconocer y fomentar la participación dentro del desarrollo de las trayectorias escolares (Fundación Chile-MINEDUC, 2013; Ochoa, 2019; Tamayo et al., 2018). A pesar de que autores como Booth y Ainscow (2012), Ordóñez-Sierra y Rodríguez-Gallego (2016) y López y otros (2018) ciertamente identifican, en dicha dimensión, un eje clave en la implementación de procesos inclusivos y de atención a la diversidad en general.

Dicho de otro modo, la evidencia empírica instala una pregunta por cuál es la relación posible, en la política inclusiva, entre participación, educación y diversidad, y cuál es la participación de la que se habla en el marco del esfuerzo institucional y democratizante de las escuelas de volverse, por mandato, inclusivas.

En base a esta evidencia, a saber, la manera como dentro del proceso inclusivo se razona un programa de inclusión y un determinado sentido y lugar, ahí, de la participación, emerge -justamente desde la participación como problema- una clave analítica que permitiría mirar no sólo "el andamiaje" del sistema inclusivo y las cuestiones que este designa, sino que también la oportunidad de hacerlo desde las versiones de la participación que la política inclusiva perfila, construye y ofrece a los sujetos.

En primer lugar, porque, de acuerdo a la evidencia, la participación efectiva aparece como pendiente. Y en segundo lugar, en tanto ofrece, desde los espacios normativos en donde no siempre se enfatiza su importancia, una caracterización de la manera en que, según Sisto (2019) se iría configurando una idea de "inclusión” específica, vigente y oficial: una 
política educativa inclusiva "a la chilena" que articula, al mismo tiempo, un tipo de participación concomitante.

Por lo tanto, ¿La participación y su aparente opacidad programática se constituye como un problema a la base del sistema escolar chileno, es un síntoma empírico no generalizable del mismo, o es el resultado del modo como el espacio escolar inclusivo se encuentra pensado y ordenado más allá de la política nacional educativa? La siguiente investigación busca precisar y entender los significados de la participación en la política educativa inclusiva para allanar algunas aproximaciones crítico-discursivas que permitan hacerse cargo de esta pregunta.

\section{Revisión de literatura}

\subsection{Política educativa, participación y democracia en la escuela}

Tomando en cuenta todo lo que la política educativa designa en términos de organización y regulación de formas programáticas de participación dentro de la escuela, no debe pasarse por alto el hecho de que esta última, en tanto que institución recientemente atravesada por un contexto de transformaciones normativas (Bellei, 2015), se articula, fundamentalmente, en virtud de determinadas modalidades administrativas que serían, en el fondo, formas políticas. En este sentido, y tomando en consideración la lectura de Foucault (1999) sobre los sistemas educativos, estos serían -incluida la escuela- el resultado de formas políticas de conservar o modificar la aprobación de los discursos, junto con los saberes y los poderes que estos implican. Vale decir, la escuela puede ser pensada como una concentración de formas de saber y modalidades de ejercicio de poder; que se puede conocer, a su vez, a través de dichas formas políticas. Las cuales, en palabras de Lechner (2002) delimitan no sólo lo que se encuentra a su disposición -digamos, lo pensable-, sino que también distribuyen el campo de lo posible, lo factible y lo deseable.

En concordancia con rastrear aquello que aparece cuando se instituye cierta idea sobre lo político, o sobre las formas que adquiere lo político, en este caso, desde la organización democrática escolar y la posibilidad inclusiva y participativa que ahí emerge, una lectura que se vuelve pertinente para poder problematizar la vigencia de esta idea y de su modalidad de implementación dentro de la escuela, se vincula con lo que Collet-Sabé (2017) propone y tensiona en torno a las lógicas que estructuran una escuela democrática.

Dicha propuesta, que podríamos denominar episódica (Belavi y Murillo, 2016) participa de una noción de democracia que también define, dentro de ella, un campo de disputa entendido como disenso en el seno de esta política educativa democrática. En donde conviven, por una parte, aquellos movimientos que descansan en la distribución jerárquica de lugares y funciones, los cuales, en el terreno de la disputa de los procesos de orden político se denominarán, de acuerdo con Rancière (2006), policía: al habilitar la puesta en juego de lógicas desigualitarias (Galende, 2019). En contraposición, otro movimiento, orientado hacia lo que el autor define como igualdad, hace referencia al proceso de verificación de la igualdad de todo ser hablante con cualquier otro. En suma, siguiendo a Rancière (2009) se define una suerte de cartografía que tiende a ordenar, dentro de lo que define y describe, distinciones, jerarquías y lugares en donde posicionar a los sujetos y las funciones que les caben (Carmona, 2019). 


\subsection{Diversidad e inclusión}

Uno de los primeros acercamientos conceptuales a la inclusión a finales de los '90 aludía a la presentación de oportunidades que hicieran concreta la demanda de universalización del acceso educativo en condiciones de equidad (UNESCO, 1990), corroborando la preocupación por eliminar, del sistema escolar, toda forma de discriminación arbitraria (MINEDUC, 2016) en razón del reconocimiento de una dignidad personal universal e incuestionable.

Ahora bien, para pensar en la inclusión como horizonte ideal de expectativas institucionales, es preciso profundizar en la definición más bien operativa de un término complejo, polisémico y a veces contradictorio (Mancebo, 2010). Situándose desde una posición que entiende la Educación como fenómeno social, es decir, como un orden de valores establecidos a partir de un sistema de relaciones, construcción y representación de un espacio social (Bourdieu, 2008), este campo se constituye como un lugar significativamente atravesado por la inclusión como exigencia normativa y aspiración programática.

La inclusión como horizonte supone el mandato o la tentativa de generar un espacio -la escuela, en este caso- susceptible de considerar en su organización a todos los sujetos posibles. Y también, de pensarlos juntos en dicho ejercicio. Vale decir, desde la inclusión se va a entender el espacio educativo como un ámbito donde puede ser verdaderamente viable incluir y mantener adheridos al sistema de escolarización y sus procedimientos, a todos los sujetos-estudiantes, independientemente de los condicionamientos diversos con que estos se muevan allí.

Existen, con base a este principio, distintas aplicaciones del término: las que, por ejemplo, enfatizan la posibilidad de que los estudiantes puedan compartir y aprender; aquellas que orientan los procesos y dinámicas de enseñanza que ocurren en la sala de clases (Pujolás, 2003); o las que apuntan al reconocimiento de una identidad diferenciada de todos, con favor a la caracterización redistributiva de una escuela debidamente particularizada por todo el alumnado que la conforma como criterio de justicia social (Fraser, 2008).

\subsection{El lugar de la participación en la inclusión educativa}

Un elemento distintivo de la definición de inclusión que Ainscow (2001) desarrolla, y que se operativiza en las sucesivas ediciones del Index for inclusion (Booth et al., 2006) del inicio, es aquél que denota y reconoce que el componente participativo fundamenta a la inclusión como proceso de mejora. En otras palabras, la participación constituye un elemento que -a diferencia del énfasis históricamente vinculado a los procesos de aprendizaje como pivotes centrales en el proceso de escolarización-, en esta definición adquiere un status articulador y constituyente dentro de andamiaje del proceso inclusivo.

Se aprecia, en este sentido, una configuración que sostiene a la participación como un asunto perfilado como un insumo independiente, paralelo, imbricado y productor de la propia inclusión o del mismo aprendizaje.

Concretamente, la participación, según Ainscow (2016) tiene que ver con la emergencia, dentro de la escuela, de un proceso de acción y de colaboración, que habilita para los implicados la posibilidad de elegir, decidir y ser reconocidos en dicho lugar. Por ende, cristaliza una entrada para caracterizar la calidad de la experiencia del alumnado en la escuela (Mendía, 2012). 
A propósito de la legitimidad y el reconocimiento que esta definición encuentra, la participación, también explicitada por la Política Nacional de Educación Especial (MINEDUC, 2005), se ajusta con precisión a la aspiración de que todos los estudiantes "reciban los beneficios de compartir el proceso de aprendizaje en un ambiente diverso" (Infante, 2007, p. 7), en consonancia con una tentativa de considerar, ahora aglutinada, la diversidad que el alumnado planteaba a la escuela.

\subsection{Participación en inclusión dentro de la política educativa}

La definición de participación que, en términos generales, MINEDUC (2009, 2013, 2015) va movilizar desde sus políticas educativas, es aquella que la entiende como una aspiración que "apunta a que todos los alumnos tengan oportunidades de participar en condiciones de igualdad en las experiencias de aprendizaje que ofrece la escuela, de convivir y pertenecer a la comunidad educativa" (MINEDUC, 2009, p. 20).

En este sentido, y dentro de los puntos de vista que corresponden a la participación como proceso, objetivo o propuesta programática, la tipificación que traza Rosa y Encina (2004) adquiere sentido en virtud de las formas que presentan las propuestas participativas al interior de la política educativa. Ambos autores proponen que la participación puede ser entendida desde dos sentidos: el primero, vinculado a la legitimación como requisito de un mecanismo efectivo de integración ("ser partícipe de"), mientras que la segunda propuesta se vincula, más bien, a al ejercicio derivado desde el poder de actuar no sólo como miembro, sino dentro de un proceso ("tomar parte de").

Desde esta perspectiva, la distinción recoge la dimensión de legitimación-reconocimiento de la participación ya descrita, junto con ilustrar cierta relación entre los dos lugares posibles dentro del proceso inclusivo: uno que valora el lugar enunciativo, y otro que, como resultado de dicho lugar, ofrece espacios de acción.

\subsection{La política pública educativa}

De acuerdo a Hodder (2000), los textos escritos en forma de leyes se consideran producciones culturales materiales que tienen como objetivo, regular normas de acción. Los cuales, de hecho, deben interpretarse en relación a contextos de producción, uso, descarte y reutilización. No resulta suficiente, entonces, considerar a los marcos legales como cuerpos independientes o descontextualizados, sino que dependen de la forma y las orientaciones que las discusiones van tomando, no sólo en los espacios institucionales de deliberación, sino en aquellos que también lo antecedieron.

Una de las acepciones que tienen los textos legales como artefactos contextuales, es su función de regulación en el abordaje de un determinado problema. Bacchi (2016) reafirma esta noción cuando plantea que algunos modelos legislativos, de hecho, visualizan a la política pública como un proceso de resolución de problemas; cuestión que determina actores relevantes en la forma en que esta modalidad resolutiva también se legitima por quienes de hecho cuentan con la atribución de generarla.

La dimensión problemática que aborda la política pública es una cuestión que, de hecho, ya menciona Foucault (Botticelli, 2015) cuando aborda la problematización como un conjunto de prácticas discursivas y no discusivas que generan que un fenómeno entre en el juego de lo que es falso o verdadero y, de esta manera, se constituya como un objeto de conocimiento. Tomándose de esta idea, Bacchi y Goodwin (2016), pensando en cómo los objetos de conocimiento son problematizados históricamente -cuándo se vuelven 
problemas- pone atención en las leyes y políticas, por cuanto sugieren puertos de entrada directos hacia esas problematizaciones. Por otro lado, los textos que cumplen con el afán de organizar una determinada normativa no sólo cristalizan como mandato un conjunto de directrices programáticas, sino que también, en palabras de Peña (2019), resultan fértiles en traslucir las divergencias que sustentan la formulación del problema que esta viene a acometer. De esta manera, es posible identificar en los textos -de acuerdo con la manera en que Paulos (2015) los sitúa como prácticas discursivas- las huellas de sus condiciones de producción.

\subsection{La escuela neoliberal como contexto}

Para el caso chileno -cuyas particularidades, cabe señalar, se inscriben en un fenómeno regional más o menos transversal- las lógicas que estructuran a la escuela se han venido fraguando hace tres décadas, en razón de una lógica que apuntaló, poco a poco, una implementación que privilegió, para articular el espacio educativo, pronunciadas condiciones de privatización (Sisto, 2019). Fue a comienzos de 1980 cuando, de acuerdo a Bellei (2015) se decretó el cambio de los proveedores de educación, trasladando esta atribución desde el gobierno central hacia los gobiernos locales (municipios) dentro del país. Lógica que al día de hoy se visualiza, también, en los modos en que se gestiona la propia inclusión como propuesta programática desde institucionalidades como el Programa de Integración Escolar (MINEDUC, 2013).

\section{Método}

Esta investigación se inscribe dentro de una aproximación cualitativa, que partirá del supuesto de la existencia de realidades complejas que se construyen, de manera dinámica, en relación a condiciones contextuales, políticas e históricas. Por esta razón, la tarea investigativa perfilada irá moviéndose, de acuerdo con Ibáñez (2006) de acuerdo al orden de los significados, sus reglas de significación, y las acciones que a través de estos se realizan.

Tomando en cuenta esta base analítica, el material a trabajar tiene que ver con el discurso, específicamente, con los productos a través de los cuales se cristaliza, y con los efectos que su ordenamiento instituye. Dicho esto, el discurso opera como una estructura que, al ser constituida por prácticas -y por ser esta misma un modo de práctica social-, permite configurar y consolidar dimensiones de lo social manifestadas, entre otras cosas, en textos. Ya desde la tradición lingüística que completa Benveniste (1997), de hecho, parte del sentido se encuentra dado por su funcionamiento textual. En consecuencia, las políticas públicas, en tanto unidades textuales, constituyen un terreno analíticamente fructífero y semánticamente prolífico de problematización de sentidos.

En palabras de Peña (2019) los textos políticos, dentro de los que ingresan las normas y políticas analizadas en este estudio, son "discursos regulados, controlados, seleccionados, con formas específicas de distribución y circulación que parecen ser la culminación de un proceso político/argumental pero que a la vez dan cuenta de otro proceso: la influencia del texto en las praxis diarias, en las vidas cotidianas de los sujetos" (p. 33). Análogamente, y como parte del trabajo con este tipo de material textual, la consideración del discurso presta atención a unidades de sentido que se manifiestan como enunciados en estructuras textuales que conciernen, al interior del material textual, a lo verbal como marco regulatorio que se vincula con contextos extra-lingüísticos; siempre resueltos a partir de 
un movimiento de remisiones entre enunciados presentes y ocultos (Paulos, 2015). En particular, este estudio se vale específicamente de algunos procedimientos del Análisis Crítico del Discurso (ACD) (Pardo, 2012) como herramienta interpretativo-analítica principal, considerando que dicha modalidad es la que mejor se ajusta en lo que respecta al discurso -y a las políticas públicas que lo contienen y producen- como fenómeno cultural, social y político. Al mismo tiempo, las perspectivas interpretativas que otorga el ACD vienen dadas por permitir desnaturalizar y problematizar producciones discursivas (Moreno, 2016). Podría afirmarse que aquí el discurso estudiado, como unidad textual cristalizada, hace referencia al momento en donde son instituidas en él lógicas que lo organizan como tal, pero que. a la vez, en una función que podríamos denominar instituyente, y en particular en el caso de los dos casos analizados, consolida formas de concebir a los sujetos y a los niveles y rangos de agencia que la política les depara.

El corpus textual seleccionado corresponde, en primer lugar, al Decreto 170/2010 que "Fija normas para determinar los alumnos con Necesidades Educativas Especiales que serán beneficiarios de las subvenciones para Educación Especial" (2010); y, en segundo lugar, al Decreto 83/2015 que "Aprueba criterios y orientaciones de Adecuación Curricular para estudiantes con Necesidades Educativas Especiales de Educación Parvularia y Educación Básica" (2015). Partiendo del supuesto de que un Decreto se asume como una unidad reglamentaria de carácter prescriptivo (Peña, 2013), ambos cuerpos legales -partes constituyentes de la política educacional inclusiva actual- son representativos de cierta forma de entender y mandatar modalidades de inclusión y participación que programan una organización normativa dentro de la escuela, pero que también constituyen, en su conjunto, unidades elegibles al sintetizar de manera detallada el carácter contingente de la política educativa: vigente en términos programáticos, operativa en términos prescriptivos, histórica en términos normativos e ilustrativa en términos paradigmáticos.

Adicionalmente, los fragmentos expuestos fueron seleccionados en virtud de su utilidad expositiva, al condensar las formas en que la participación aparece declinada -o difractada- por lo que se configura dentro de la política como inclusión. Del mismo modo, la forma en que la inclusión provee modalidades institucionales de participación expresa cómo, al interior de la política inclusiva, también se yuxtapone un horizonte participativo por derecho propio: un discurso dependiente pero autónomo en torno a lo que perfila la participación y sus formas en la política chilena.

Para la realización del análisis propiamente tal, el trabajo recurre a micro-estrategias que, de acuerdo al modelo de Pardo (2007) consisten en: a) la identificación de metonimias entendidas como figura que traslucen modelos culturales subyacentes; b) develamiento de operaciones de énfasis y procesos de asociación generativa visibles en virtud de la integración, reordenamiento o sustitución de unidades textuales y c) identificación de procesos intertextuales referentes a relaciones contextuales que incluyen la situación socio-cultural y los marcos políticos donde se inscriben las prácticas discursivas.

\section{Resultados}

\subsection{La inclusión sin participación sigue siendo inclusión. Los énfasis de la política}

En primer lugar, la participación ocupa un lugar jerarquizado que no sólo figura, en relación a la inclusión, dentro del texto, sino que dicha posición es instituida por la forma 
en que los criterios del Index for inclusion son recogidos por las interpretaciones prescriptivas institucionales. Dicha participación es el resultado de posiciones discursivas que instituyen parámetros de relevancia que denotan y dan cuenta de los procedimientos inclusivos que cada norma, en tanto cuerpo legal, persigue y genera. Veamos un caso tomado del Decreto 170:

Cuando la evaluación diagnóstica multiprofesional confirme la presencia del trastorno y éste afecte significativamente el aprendizaje escolar y/o la participación del o la estudiante en la escuela de acuerdo a lo establecido en el artículo 10 del presente reglamento. (Decreto 170, 2010, p.17)

Dentro del ordenamiento de la política educativa que tipifica este Decreto en particular, participación y aprendizaje -en este caso, su afectación por presencia de un trastornoaparecen como dos significantes que se organizan bajo un orden de semejanza prescriptiva: se enuncian al unísono porque se desprende de ambos la misma relevancia programática. El "compromiso" que identifica el diagnóstico del déficit implica, en él o la estudiante, afectar ambas condiciones. Por ende, si atendemos a la noción inclusiva que recoge la política de manera mayoritaria, el fragmento no sólo corresponde y concuerda con dicha idea, sino que parece desprenderse de ella de modo casi literal. Colocando, a la altura de un mismo régimen de importancia, ambas cualidades como si fuesen componentes conceptuales equitativos, participantes de un mismo fenómeno: el diagnóstico como requisito fundante de la atención a la diversidad.

De acuerdo a la manera en que la política educativa comprende esta tarea, la idea concuerda con lo que López y otros (2014) establecen en torno al modelo de integración que supone la política educativa que se maneja en el Decreto 170: que emparenta la lógica de los derechos humanos con una que se desprende de modelos de naturaleza médicoclínica. Al mismo tiempo, López y otros (2014) justifica la aparición de este procedimiento normativo por la necesidad que surge de plantear una atención individual de la discapacidad, debido a que el proceso diagnóstico permite determinar el déficit de un sujeto específico (Apablaza, 2018) beneficiario de la ayuda especializada y convocado ontológicamente como tal.

Ahora bien, lo interesante no es necesariamente esta construcción discursiva -que más allá de su utilidad en corroborar la presencia de la racionalidad propia del Index for inclusion, en la literatura especializada también ya se identifican dichos componentes en su conceptualización (Echeita, 2006; López, 2014)- sino el modo en que esta construcción, anclada en lo que se llamará régimen de relevancia, irá variando sus énfasis en virtud del modo en que supuestamente se irá perfilando una idea de inclusión que no siempre es tan constante como podría suponerse desde su declaración de principios. Otro fragmento, del mismo Decreto, ilustra esta idea:

Provisión de medios y recursos materiales educativos que faciliten la participación, la autonomía y progreso en los aprendizajes de los y las estudiantes. (Decreto 170, 2010, p. 30)

En efecto, esta relación de correspondencia y complementariedad conceptual entre participación-aprendizaje dará lugar a cierto desplazamiento que configurará una nueva jerarquía emergente, cuyo efecto radica en que la participación va a ser entendida discursivamente como un insumo del aprendizaje. Pasando de una inclusión entendida desde estos dos ejes constitutivos, orientados desde el mismo régimen de relevancia, y codependientes en su implicación a un proceso de permanente elaboración, hacia la subsidiariedad de un concepto que trabaja al servicio de otra cosa que antes más bien 
constituía. En otras palabras, la participación pasa, de formar a la inclusión, a formar al concepto que sí es formativo del proceso inclusivo.

\subsection{La participación como síntoma}

Partiendo de la base que la inclusión, en cierto modo, es una forma de otorgar y distribuir los lugares y grados de reconocimiento de los sujetos que se benefician de ella, es relevante precisar cómo se dan estas operaciones y -más aun- cuáles son los sujetos que se desprenden de este proceso de legitimación distributiva de lugares y funciones. En el fondo, y en palabras de Rancière (2018) cuál es lugar que se les asigna, y a qué supuestos responde la naturaleza de dicho reparto.

Para comenzar, conviene revisar una de las claves que puntualiza el Decreto 170, en relación a los procedimientos de evaluación diagnóstica a los cuales debe ser sometido un estudiante, eventualmente beneficiario de las ayudas psicosociales que el dispositivo inclusivo -referido aquí como integrativo- estaría en condiciones de brindarle:

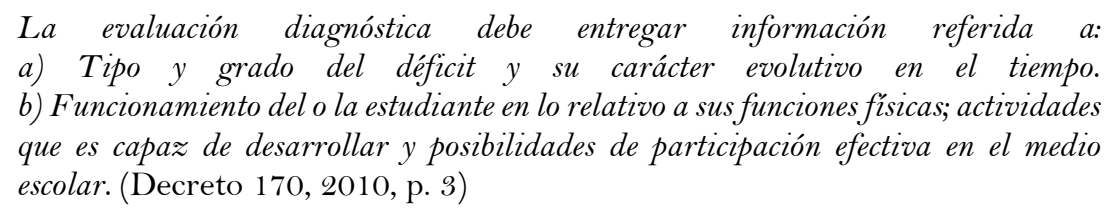

En efecto, la participación es, primero que todo, un signo: una especie de dato a consignar. Vale decir, un elemento susceptible y necesario de ser pesquisado como un criterio más en el diagnóstico del déficit de un sujeto que emerge producto de él. Por lo tanto, su lugar es patente y explícitamente referido como un asunto eminentemente semiótico-descriptivo, que forma parte de un procedimiento de averiguación que arrojará datos sobre un sujeto "en condiciones de ser incluido" como resultado de dicha investigación. Ahora bien, el uso que comporta la participación en dicho proceso existe, además, como un parámetro que la circunscribe exclusivamente a las posibilidades que el estudiante pueda tener a propósito de la envergadura de su propio déficit.

La participación, perfilada aquí como un criterio funcionalista, aporta entonces a la distinción esclarecedora del eje habilidad/inhabilidad. Sin embargo, en relación al proceso evaluativo, este sujeto, ¿A qué se encuentra habilitado?, pues a formar parte de las actividades que la escuela o el currículum le propone. Entonces, cuando la participación aparece vinculada al mismo nivel de alguna característica sintomática constitutiva del déficit de un sujeto, es decir, cuando se configura, con la norma, como una suerte de participación-habilitante, nuevamente se le subordina. En este caso, a otra categoría que sí sería una garantía de reconocimiento: dar con un diagnóstico. En virtud de este procedimiento riguroso de simplificación sintomática, en el fondo, de parametrización normativa de una acción ético-política, participación y aprendizaje, como en el punto anterior, sí se emparentan: sólo que en virtud de un diagnóstico que se ha vuelto garantía de inclusión, y que, nuevamente, la mediatiza.

En consecuencia, este uso de la participación como un sustrato más del diagnóstico, contribuye a perfilar un sujeto a quien se le observará en razón de un "medio escolar" disponible que habitualmente se circunscribe al currículum que, por ejemplo, el Decreto 83 propone flexibilizar como acción inclusiva. Al mismo tiempo, el proceso diagnóstico instala una dimensión "probabilística" del sujeto participador que, contraviniendo el afán objetivizante que tendría una taxonomía de las distintas dificultades del estudiante, aquí demanda encontrarle "posibilidades de participación". La participación es un dato 
diagnóstico, pero también un gradiente que articula modos de hacerse posible: porque permanece al servicio de mecanismos discursivos que precisan desagregarla en virtud de lo que desean implicar.

Así, del mismo modo en que se entiende la participación como instrumento o insumo, también aparecen otras formas que semantizan aquello que esta buscaría implicar en términos concretos: ¿Qué es, si no otra cosa, el que la participación pueda ser una posibilidad, una probabilidad o una condición eventualmente susceptible de ser evaluada como si se tratase de signos de un déficit ponderable diagnósticamente? En este sentido, la posibilidad de participar es una construcción discursiva dicotómica "lo logra" o "no lo logra" afín al diagnóstico, en donde esta "probabilidad de participación” es una operación discursiva que inscribe en ella grados posibles dentro de un ámbito de acción disponible, pero no siempre del todo materializado en términos prescriptivos, ya que, ¿Cómo se objetiva o se hace inteligible esa participación supuestamente inexistente? En parte debido a esa categoría que la presenta en términos sintomatológicos que ponderan ahí un déficit.

Mientras tanto, el devenido síntoma participativo es un aparato que le sirve a la búsqueda diagnóstica para definir un tipo de sujeto en donde esta desagregación pueda tener efecto y, por cierto, utilidad metodológica. Más allá de lo que se podría esperar que esta pueda implicar en términos de posibilidad, actividad concreta o atributo inclusivo, la participación queda resuelta ahí: se termina en el déficit que la encuentra.

Por ejemplo, dicha reconversión de la participación "como posibilidad", que por cierto también entra sin mucho esfuerzo en la lógica instrumentalizada, da cuenta de un procedimiento metonímico particular: la idea de que "las posibilidades de participación" requieren de un estado pre-participativo que es necesario conocer de antemano. Y que, en cierto sentido, justificará el ámbito de participación donde esta pueda ser perceptible y garantizada. ¿Cuál es la naturaleza del sujeto se desprende de esta conclusión? Pues que existe, de hecho, un sujeto ontológico individual que sólo participa cuando se le oferta la instancia, por ejemplo, diagnóstica, de encontrarse con un otro.

\subsection{Participar de las evaluaciones: un lugar disponible}

Por otra parte, uno de los asuntos que permite encontrar a la participación como modalidad de reconocimiento, dice relación con un componente estrictamente cuantitativo y muy sintonizado con lo que Cornejo (2006) reconoce como distintivo en un sistema educativo como el chileno:

\section{El Ministerio de Educación deberá hacer las adecuaciones necesarias para que los alumnos con NEE puedan participar en las mediciones de la calidad de la educación. Por ende, la adecuación curricular favorece la participación en los procesos de evaluación. (Decreto 83, 2015, p. 19)}

En primera instancia, el fragmento recogido del Decreto 83 sitúa la participación como una condición necesaria para hacer otras cosas: en este caso, constituir al estudiante en tanto sujeto de medición. Esta referencia es fundamental, puesto que se encuadra dentro de la necesidad del Estado de almacenar y suministrar información para distribuir los recursos que se destinan a los dispositivos tecnificados que hacen participar a los sujetos desde la lógica de la subvención. Por lo tanto, las evaluaciones permitirían, en principio, perfilar al estudiantado que se beneficia de los marcos normativos inclusivos/subsidiarios.

Esta configuración, a primera vista atravesada a cabalidad por una racionalidad instrumental pero también económica, tiene dos lecturas posibles: a) una asociada a la 
comprensión del individuo en tanto entidad cuantificable y de la política pública como la manera de conferirles una entidad que, por el hecho de hacerlos ponderables, los comprende como sujetos en la medida que conoce y aporta en su caracterización, y, b) la posibilidad de instrumentalizar el déficit -y la misma participación- en la medida que sustenta criterios para poder incluir al sujeto a los mecanismos de cuantificación y estandarización de procesos, más que a realizar algún tipo de convocatoria que exceda la medición y que legitime la necesidad de los individuos de ser, por ejemplo, sujetos de participación. Y no sólo de las evaluaciones, sino de aquello que las evaluaciones muchas veces perderían de vista: en este caso, las razones que inciden y articulan la necesidad de que se pueda comprender distinto esta manera de participar que, a ojos del Estado, habitualmente aparece como necesitada de subsidio para la escuela.

Otro asunto decisivo se desprende de esta implicación: las adecuaciones "permiten participar de las mediciones". Junto con la incorporación del sujeto a los procedimientos de medición, la posibilidad de adecuar el currículum transforma nuevamente el significado de la participación en un indicador que documenta una exclusión previa, pero que al mismo tiempo justifica ese mecanismo. Cuando se asume la necesidad de adecuar, se está reconociendo que la participación estaba preferentemente -o por completo- anclada al procedimiento de medición que antes no incluía a algunos estudiantes ahora diagnosticables, al mismo tiempo que corrobora una idea valorativa y responsabilizante de este proceso en ese alumnado. Es nuevamente, el problema de cierta inclusión: debido a antes excluía, ahora incluye; pero corre el riesgo de excluir a propósito de la gramática operativa que pone en juego en el proceso.

En el fondo, el reconocimiento del Decreto cierra e individualiza, como también curriculariza las posibilidades de participación, a partir de la traducción inclusiva que se realiza en la configuración de este sujeto de medición. Y de paso oblitera la pertinencia de una pregunta por aquellos fenómenos que generaban que, antes de la adecuación, los sujetos no pudiesen ser evaluados. Finalmente, por la evaluación o el currículum, los sujetos son dignatarios y susceptibles de ser hechos parte de la participación, mucho más que de hacerse partícipes en ella. La distinción, que no es casual ni del todo circunstancial, formula un determinado tipo de sujeto al momento de reconocerlo desde la dimensión participativa.

\subsection{Tres evidencias de la participación: Objetada, tutelar y paradójica}

Una de las principales características que adquiere la participación dentro de la política inclusiva es su carácter objetado por las nociones que conviven y dialogan con las pretensiones implicativas que esta adquiere dentro del texto. En este sentido, la participación como modalidad inclusiva, surge como resultado de procesos de condicionamiento que la sustraen de sus pretensiones inclusivas originales para, en su mayoría, lograr resituarla con éxito.

En el análisis se revela, desde la identificación de los distintos sentidos de la participación, una naturaleza habitualmente tutelada o encapsulada dentro de la política inclusiva. Esta distinción -que se constata más frecuentemente en aquellas declinaciones mayormente vinculadas a su lugar como objetivo programático- tiende a ser la modalidad principal desde la cual toman sentido las nociones participativas dentro del texto y en lo relativo a sus efectos. 
En tercer lugar, y en una lógica que diverge de las lecturas que condicionan las orientaciones que toma dentro del proceso inclusivo, emerge dentro de la política una participación de carácter paradojal que aparece de manera transversal en la política, imbricando gran parte de sus prescripciones y efectos. Efectivamente, y pese a que las mencionadas declinaciones a las cuales se someten sus sentidos disponibles aparecen en general ancladas a los objetivos de la política inclusiva, al mismo tiempo, tanto el concepto como su centralidad interpretativa permiten esbozar una dimensión controvertida de la misma, que, de hecho, ya se advierte cuando Rancière (Belavi y Murillo, 2016) somete a escrutinio los supuestos de la escuela democrática como dominio que articula, desde el consenso, racionalidades policiales e igualitarias que pugnan en torno a la igualdad(o la versión de igualdad que instituye una inclusión institucional).

\section{Discusión y conclusiones}

A través del análisis crítico al que se somete el corpus que perfila la política educativa inclusiva, es posible reconocer la presencia de tres discursividades estructurantes, estratégicas y fundamentales que orientan la manera en que la participación es declinada y asignada dentro de la política educativa, y que resultan plenamente consistentes con la forma en que el discurso de la inclusión mediatiza divergencias junto con producir expresiones de aquello que se encuentra imbricado en ella.

Una primera discursividad que emerge del análisis alude a la dimensión neoliberal que constriñe e impacta el andamiaje completo de la política educativa. De hecho, más allá de constituirse en un mero condicionamiento, lo que esta configura tiene relación con su condición paradigmática, orientadora del sentido mismo de los lineamientos y maneras de comprender lo que la política educativa, la participación y sus supuestos persiguen en los distintos ámbitos en donde esta última aparece visible. Partiendo de la base de que los procesos privatizadores canalizan la gestión hacia procesos de maximización del rendimiento (Falabella, 2015), personalización del déficit como estrategia de gestión y razonamiento individualizado de las dinámicas escolares, la noción de participación y sus posibilidades se encuentran indefectiblemente atravesadas por esta discursividad de manera constitutiva.

Una segunda discursividad que opera en el modo de comprender y de orientar los sentidos de la participación y, sobre todo, de la inclusión como propuesta programática, alude a la racionalidad científico-técnica constitutiva de la institucionalidad educativa en torno a la gestión de la diferencia (Matus y Rojas, 2015). Visible, por ejemplo, en la incorporación de dispositivos especializados que se plantean el abordaje del problema que viene a intervenir como parte de la noción operativa de la inclusión, o cuando subordinan a la participación al aparataje diagnóstico que la supone como otro indicador del proceso evaluativo integral.

Concretamente, parte de la investigación que problematiza este fenómeno a propósito de nociones críticas como la individuación del déficit en virtud de "tecnologías psi” o la determinación de categorías diagnósticas dentro de la escuela como parámetro informativo y de ajuste gubernamental (Apablaza, 2015), se conforman procesos que sintonizan -y por cierto programan- un tipo de participación oficial que, de hecho, se incluye como tecnología que vertebra, desde una lógica instituida, algunos de estos propósitos. 
Finalmente, la tercera discursividad operativa y paradigmática alrededor de la inclusión y de los sentidos de la participación, se vincula con la noción de Derechos, que viene a justificar, por parte la institucionalidad, la incorporación de la inclusión como respuesta programática para la gestión de la diferencia dentro de la escuela. La inclusión, articulada desde el Derecho, aparece como una tarea que demanda marcos normativos y planes de ejecución especializada que resuelvan la tarea pendiente que esta demanda pone en evidencia y que la política inclusiva recoge cuando instituye mandatos de acomodación curricular, o bien cuando designa sujetos medibles que participan de la matriz inclusiva que los decretos tipifican. De cierto modo, la política inclusiva per se responde al interés democrático que elabora garantías, salvo que la modalidad particular de organización de la política justifica, ciertamente, entradas analíticas a los sentidos que dispone.

Tomando en cuenta la manera como se anudan estas racionalidades al interior de la política, dentro de las cuales poner atención a la participación revelaría la manera en que se ordenan, una de las derivas que propone este análisis revelaría el potencial político de pensar a la participación como una fuerza que, simultáneamente, forma parte de un orden único al cual contribuye de manera ambivalente, donde no debe perderse de vista que su problematización, al menos, desliza una posibilidad de participar en la configuración de las refutaciones de los sentidos que la restringen. Una opción disponible, entonces, parte por pensar cómo esta organización indica las formas en que se razonan los escenarios normativos en los cuales las políticas habilitan lugares y distribuyen, en definitiva, rangos de acción, márgenes de agencia y porciones de posibilidad: a propósito de lo que las políticas inauguran como espacio de discusión sobre los límites de lo posible.

A la luz de acontecimientos psicosociales recientes que interpelan "las normas políticas, jurídicas y cívicas que fundamentan la regulación de la vida en común” (Araujo, 2016, p. 9 ), pensar exhaustiva y críticamente en los pivotes de la participación constituye una alternativa para ponderar cualitativamente las condicionantes históricas de un proceso esquivo y acaso todavía pendiente para la política educativa y la institucionalidad participativa en general. Participar de las nuevas maneras de pensarla, entonces, no es otra cosa que hacerse en esa participación posible. Al fin y al cabo, porque tomar parte es hacerse de un lugar: en la restitución del ser con otros; al interior de la gran discusión que surge cuando pensamos en conjunto sobre el orden que decidimos que nos convoque.

\section{Referencias}

Ainscow, M. (2001). Desarrollo de escuelas inclusivas: Ideas propuestas y experiencias para mejorar las instituciones escolares. Narcea.

Ainscow, M. (2016). Diversity and equity: A global education challenge. New Zealand Journal of Educational Studies, 51, 143-155. https://doi.org/10.1007/s40841-016-0056-x

Apablaza, M. (2018). Inclusión escolar, marginación y apartheid ocupacional: Análisis de las políticas educativas chilenas. Journal of Occupational Science, 25(4), 3-15. https://doi.org/10.1080/14427591.2018.1487260

Apablaza, C. (2015). El orden en la producción de conocimiento: Normatividades en la educación chilena en torno a diversidad. Estudios pedagógicos, 41, 253-266. https://doi.org/10.4067/S0718-07052015000300016

Araujo, K. (2016). El miedo a los subordinados, una teoría de la autoridad. LOM. 
Bacchi, C. (2016). Problematizations in health policy: Questioning how "problems" are constituted in policies. SAGE Open, 6(2), 1-16. https://doi.org/10.1177/2158244016653986

Bacchi, C. y Goodwin, S. (2016). Poststructural policy analysis: A guide to practice. Macmillan.

Belavi, G. y Murillo, F. (2016). Educación, democracia y justicia social. Revista Internacional de Educación para la Justicia Social, 5(1), 13-34.

Bellei, C. (2015). El gran experimento: Mercado y privatización de la educación chilena. LOM.

Benveniste, E. (1997). Problemas de lingüística general I. Siglo XXI.

Booth, T. y Ainscow, M. (2012). Index for inclusion. Guía para la evaluación y mejora de la educación inclusiva. Consorcio Universitario para la Educación Inclusiva.

Booth, T., Ainscow, M., Black-Hawkins, K., Vaughan., M. y Shaw, L. (2002). Index for inclusion: Developing learning and participation in schools. Centre for Studies on Inclusive Education.

Booth, T., Ainscow, M. y Kingston, D. (2006). Index para la inclusión: Desarrollo del juego, el aprendizaje y la participación en la educación infantil. Centre for Studies on Inclusive Education.

Booth, T., Simón, C., Sandoval, M., Echeita, G. y Muñoz, Y. (2015). Guía para la educación inclusiva. Promoviendo el aprendizaje y la participación en las escuelas. REICE. Revista Iberoamericana sobre Calidad, Eficacia y Cambio en Educación, 13(3), 5-19.

Botticelli, S. (2015). La gubernamentalidad del estado en Foucault: Un problema moderno. Praxis Filosófica, 42(1), 83-106.

Bourdieu, P. (2008). Capital cultural, escuela y espacio social. Siglo XXI.

Carmona, J. (2019). La política no es nomos sino nemeïn: Sobre el sentido del reparto de lo sensible en J. Ranciére. En F. Vega y V. Rocco (Eds.), Estética del disenso: Políticas del arte en Jacques Rancière (pp. 23-42). Doble Ciencia.

Cornejo, R. (2006). El experimento educativo chileno 20 años después: Una mirada crítica a los logros y falencias del sistema escolar. REICE. Revista Iberoamericana sobre Calidad, Eficacia y Cambio en Educación, 4(1), 118-129.

Collet-Sabé, J. (2017). Escoles democràtiques en comú. Primeres notes per produir escoles democràtiques a partir de pràctiques comunitzants. Pedagogia i Treball Social, 6(2), 32-54. https://doi.org/10.33115/udg_bib/pts.v6i2.22150

Echeita, G. (2006). Educación inclusiva o educación sin exclusiones. Narcea.

Falabella, A. (2015). El mercado escolar en Chile y el surgimiento de la nueva gestión pública: El tejido de la política entre la dictadura neoliberal y los gobiernos de la centroizquierda (1979 a 2009). Educação y Sociedade 36(132), 699-722.

https://doi.org/10.1590/ESo101-73302015152420

Foucault, M. (1999). El orden del discurso. Tusquets.

Fraser, N. (2008). La justicia social en la era de la política de identidad: Redistribución, reconocimiento y participación. Revista de Trabajo, 4(6), 83-99.

Fundación Chile, Centro de Innovación en Educación y MINEDUC (2013). Análisis de la implementación de los programas de integración escolar (PIE) en Establecimientos que han incorporado estudiantes con necesidades educativas especiales transitorias (NEET).

Galende, F. (2019). Rancière: El presupuesto de la igualdad en la política y en la estética. Eterna Cadencia.

Hodder, I. (2000). The interpretation of documents and material culture. En N. Denzin y Y. Lincoln (Eds.), Handbook of qualitative research (pp. 393-402). SAGE. 
Ibáñez, J. (2006). Presentación. En M. Canales (Ed.), Metodologías de investigación social: Introducción a los oficios (pp. 11-28). LOM.

Infante, M. (2007). Inclusión educativa en el cono sur: Chile. UNESCO.

Lechner, N. (2002). Las sombras del mañana: la dimensión subjetiva de la política. LOM.

López, V., Gónzález, P., Manghi, D., Ascorra, P. y Oyanedel, J. (2018). Políticas de inclusión educativa en Chile: Tres nudos críticos. Archivos Analíticos de Políticas Educativas, 157(26), 1-24. https://doi.org/10.14507/epaa.26.3088

López, V., Julio, C., Morales, M., Rojas, C. y Pérez, M. V. (2014) Barreras culturales para la inclusión: Políticas y prácticas de integración en Chile. Revista de Educación, 363, 256-281.

Mancebo, M. (2010, abril). La inclusión educativa: un paradigma en construcción. IV Encuentro Internacional de investigadores de políticas educativas. Argentina.

Matus, C. y Rojas, C. (2015). Normalidad y diferencia en nuestras escuelas: A propósito de la Ley de Inclusión Escolar. Docencia, 56, 47-56. https://doi.org/10.4067/S07 18-07052015000300009

Mendía, R. (2012). El aprendizaje-servicio como una estrategia inclusiva para superar las barreras al aprendizaje y a la participación. Revista Educación Inclusiva, 5(1), 71-82.

MINEDUC. (2005). Nueva política de educación especial: Nuestro compromiso con la diversidad. MINEDUC.

MINEDUC. (2009). Criterios y orientaciones de flexibilización del currículum: Para dar respuesta a la diversidad en los distintos niveles y modalidades de enseñanza. MINEDUC.

MINEDUC. (2013). Programa de integración escolar PIE, decreto supremo $N^{\circ} 170$ de 2009: Manual de orientaciones y apoyo a la gestión (Directores y Sostenedores). MINEDUC.

MINEDUC. (2015). Decreto $N^{\circ}$ 83/2015: Aprueba criterios y orientaciones de adecuación curricular para estudiantes con necesidades educativas especiales de educación parvularia y educación básica. MINEDUC.

MINEDUC. (2016). Programa de integración escolar, PIE, ley de inclusión 20.845: Manual de apoyo a la inclusión escolar en el marco de la reforma educacional. MINEDUC.

MINEDUC. (2019). Comunidades educativas inclusivas. Claves para la acción. MINEDUC

Moreno, E. (2016). El análisis crítico del discurso en el escenario educativo. Zona Próxima, 25, 129148. https://doi.org/10.14482/zp.22.5832

Ochoa, A. (2019). El tipo de participación que promueve la escuela, una limitante para la inclusión. Alteridad, Revista de Educación, 14(2), 184-194. https://doi.org/10.17163/alt.v14n2.2019.03

Ordóñez-Sierra, R. y Rodríguez-Gallego, M. (2016). Los grupos interactivos como metodología didáctica en educación secundaria: Estudio de casos en un centro constituido en comunidad de aprendizaje. Revista de Investigación en Educación, 14(2), 141-155.

OECD. (2018). Education policy outlook 2018: Putting student learning at the centre. OECD Publishing. https://doi.org/10.1787/9789264301528-en

UNESCO. (1990). Declaración mundial sobre educación para todos y marco de acción para satisfacer las necesidades básicas de aprendizaje. UNESCO.

Pardo, N. (2012). Análisis crítico del discurso: Conceptualización y desarrollo. Cuadernos de Lingüística Hispánica, 19, 41-62.

Pardo, N. (2007). Cómo hacer análisis crítico del discurso: Una perspectiva latinoamericana. Frasis. 
Paulos, D. (2015). El discurso y su relación con el límite exterior del lenguaje. Cinta de Moebio, 53, 190-204. https://doi.org/10.4067/So7 17-554X2015000200007

Peña, M. (2013). Análisis crítico de discurso del Decreto 170 de subvención diferenciada para necesidades educativas especiales: El diagnóstico como herramienta de gestión. Psicoperspectivas, 12(2), 93-103.

Peña, M. (2019). El análisis crítico de discurso en textos de políticas públicas: Lineamientos para una praxis investigativa. La Trama de la Comunicación, 23(1), 31-46. https://doi.org/10.35305/lt.v23i1.678

Popkewitz T. (2018). Reform and making human kinds: The double gestures of inclusion and exclusion in the practice of schooling. En E. Hultqvist, S. Lindblad y T. Popkewitz (Eds.) Critical analyses of educational reforms in an era of transnational governance. Educational governance research (pp. 157-197). Springer. https://doi.org/10.1007/978-3-319-61971-2_8

Pujolás, P. (2003). Aprendre junts alumnes diferents. Els equips d'aprenentatge cooperatiu a l'aula. Eumo Editorial.

Rancière, J. (2006). Policía, política, democracia. LOM.

Rancière, J. (2009). El reparto de lo sensible: estética y política. LOM.

Rancière, J. (2018). El maestro ignorante: cinco lecciones sobre emancipación intelectual. Hueders.

Rosa, M. y Encina, J. (2004). Oralidad y participación. De cómo trabajar las historias orales desde la investigación participativa. Introducción a las historias orales de Pedrera. En J. Encina, J. Pino, F. Sierra y M. Rosa (Eds.), Participación, comunicación y desarrollo comunitario. Democracias participativas 1, (pp. 229-273). Editorial Atrapasueños.

Sandoval, M., López, M., Miquel, E., Durán, D., Giné, C. y Echeita, G. (2002). Index for inclusion, una guía para la evaluación y mejora de la educación inclusiva. Contextos Educativos, 5(1), 227-238.

Sisto, V. (2019). Inclusión “a la chilena”: La inclusión escolar en un contexto de políticas neoliberales avanzadas. Archivos Analíticos de Políticas Educativas, 27(23), art 5.

https://doi.org/10.14507/epaa.27.3044

Tamayo, M., Carvallo, F., Sánchez, M., Besoaín-Saldaña, A. y Rebolledo, J. (2018). Programa de integración escolar en Chile: Brechas y desafíos para la implementación de un programa de educación inclusiva. Revista Española de Discapacidad, 6(1), 161-179.

\section{Breve CV del autor}

\section{Claudio Herrera}

Psicólogo clínico de la Universidad de Santiago de Chile, Magister en Psicología, mención Psicología Social de la Universidad Diego Portales. Con trayectoria profesional vinculada al ámbito educativo como parte del Programa de Integración Escolar, y actualmente desde el trabajo como docente semi-presencial a tiempo parcial en la Universidad UNIACC, y como docente asociado en la Universidad Bernardo O’Higgins desde la especialidad Social y Comunitaria aplicada a la formación en pregrado de las carreras de Trabajo Social y Psicología, sus líneas de investigación actuales incluyen la escuela y sus lógicas programáticas, las políticas educativas, los métodos cualitativos de investigación, las modalidades de intervención social $\mathrm{y}$ los estudios discursivos. ORCID ID: https://orcid.org/OOOO-0001-8880-9696. E-mail: claudioherrera@docente.ubo.cl 